\title{
ÉCRIRE FACE À LA « FAILLE DIASPORIQUE" » : HAÏTI KENBE LÀ ! (2010) DE RODNEY SAINT-ÉLOI ${ }^{1}$
}

\author{
Alessia ViGNOLI \\ Université de Varsovie
}

\begin{abstract}
En): The first wave of Haitian migration in Quebec dates back to the 1960s, as a direct consequence of François Duvalier's dictatorial regime (1957-1971). The works published in Montreal by Haitian writers such as Anthony Phelps, Gérard Étienne and Émile Ollivier were written in a state of urgency and were strongly connected to the "duty of memory" and the necessity of bearing witness to the horrors of the Duvalier dictatorship. After the earthquake that struck Haiti in January 2010, some Haitian writers living in Quebec felt compelled to write in order to cope with the disaster. Soon after the publication of Dany Laferrière's text Tout bouge autour de moi (2010) the founder of the publishing house "Mémoire d'encrier" Rodney SaintÉloi published Haïti kenbe là ! ("Haiti rise up!", 2010). In his account of the aftermath of the earthquake Saint-Éloi, who survived the disaster, reflects on his diasporic situation. The aim of this essay is to analyze the sense of belonging to two cultures and two countries, Haiti and Quebec, as it emerges from the reading of Haïti kenbe là !. In an attempt to face this feeling intensified by the disaster, Saint-Éloi finds in literary creation the only possible answer to the "inner fault" that haunts the Haitian writer living in Quebec.
\end{abstract}

Keywords (En): post-earthquake literature ; Haitian diaspora in Québec ; Rodney Saint-Éloi ; disaster writing; literature of urgency.

Mots-clés (Fr) : littérature post-sismique ; diaspora haïtienne au Québec ; Rodney Saint-Éloi ; littérature de la catastrophe ; écriture de l'urgence.

\section{Introduction}

Les œuvres des écrivains haïtiens exilés au Québec à partir du début des années 1960, à la suite des exactions commises par le régime dictatorial de François Duvalier (1957-1971), sont marquées par le besoin de témoigner et d'assumer le « devoir de mémoire $»^{2}$ à l'égard des expériences traumatisantes et de l'oppression vécues par la société haïtienne. On le voit bien dans les romans Moins l'infini (1973) et Mémoire en colin-maillard (1976) d'Anthony Phelps, Le Nègre crucifié (1974) de Gérard Étienne ou dans les nouvelles qui composent Paysage de l'aveugle (1977) d'Émile Ollivier. Ces fictions qui voient le jour au Québec dans les années 1970 peuvent être définies comme « interventionnistes » parce qu'elles représentent un véritable acte de dénonciation de la situation collective haïtienne sous le duvaliérisme. Cet acte est encouragé par la position diasporique des auteurs concernés qui les libère des contraintes et des (auto)censures. Dans ces textes,

\footnotetext{
${ }^{1}$ Le présent article a été réalisé grâce à la subvention du projet Literackie obrazowanie katastrof naturalnych $w$ prozie haitańskiej $i$ antylskiej ( $\mathrm{n}^{\circ}$ 2017/27/N/HS2/00671) accordée par Narodowe Centrum Nauki, Polska (Centre National de Recherche Scientifique, Pologne).

${ }^{2}$ Concept utilisé en référence au génocide du peuple juif et au système concentrationnaire nazi (LALIEU, $2001: 83$ ).
} 
l'entrecroisement de la fiction et du témoignage est très présent, au point qu'il est parfois difficile de faire une distinction entre l'écrivain et le narrateur.

À cause de leur statut d'exilés politiques, les écrivains de la première vague d'immigration haïtienne se rapprochent des écrivains québécois, en particulier de leurs prises de position qui revendiquent, à la même période, l'affirmation collective des «Nègres blancs d'Amérique », pour reprendre le titre de l'essai de Pierre Vallières publié en 1968. Józef Kwaterko constate une convergence entre les romans des premiers écrivains immigrés haïtiens et les romans québécois des années 196080. Il estime que ces deux corpus, haïtien et québécois, sont « [...] travaillés, sur le plan thématique global, par les problèmes de la fragilisation identitaire et de la déprise historique » (KWATERKO, 2002 : 215). Les similitudes ne s'arrêtent pas au plan thématique. Elles se retrouvent au niveau plus profond des structures énonciatives et des techniques de narration. KWATERKO $(2002: 215)$ remarque à ce propos « $[\ldots]$ une pulsion similaire à la fragmentation $[\ldots]$ au récit éclaté, à l'enchâssement des voix multiples, au monologue intérieur, à tous les dispositifs énonciatifs brisés qui creusent le sentiment de l'aliénation et du déracinement ».

Les écrivains haïtiens des générations suivantes, confrontés davantage au pluralisme culturel de Montréal, vont assumer le «sentiment de double appartenance » dont parle l'auteure haïtienne Yanick Lahens dans son essai L'Exil : entre l'ancrage et la fuite, l'écrivain haïtien. Ils abandonnent l'écriture militante de leurs aînés, ancrée dans le politique, et abordent sur un mode plus subjectif la question de l'identité et de l'exil. Cette tendance est propre à la génération des écrivains haïtiens qui sont arrivés à Montréal dans leur jeunesse. Le déchirement entre Haïti et le Québec en tant que topos central de l'écriture s'efface chez eux au profit d'une mise en place des espaces pluriels et hybrides, ce qui favorise une écriture ludique, mélangeant les genres et les mémoires, ouverte à de nouvelles expériences. Comme le dit Yanick LAHENS (1990: 59), «[1]es écrivains les plus jeunes convertiront l'exil en errance et nomadisme. Ils ont baigné plus tôt dans la culture urbaine des métropoles, dans la rumeur des grandes villes. [...] l'origine continuera à les hanter mais sur un mode différent ». Ce sera surtout le cas de Dany Laferrière (Comment faire l'amour avec un nègre sans se fatiguer, 1985) et Stanley Péan (Zombi blues, 1996).

Or, suite au tremblement de terre qui a frappé la capitale haïtienne Port-auPrince et d'autres villes du pays caribéen le 12 janvier 2010, certains auteurs haïtiens du Québec se sentent de nouveau obligés de témoigner pour faire face à la tragédie. Marie Estripeaut-Bourjac utilise l'expression « écriture de l'urgence » en tant que sous-genre de l'écriture testimoniale pour étudier l'emploi du témoignage en Amérique latine. Elle estime que la visée des diverses formes textuelles d' « écriture de l'urgence» (autobiographie, mémoires, journal intime, chronique, récit de voyage) est de «[...] favoriser la création de solidarités et la demande d'appuis, nationaux et internationaux » (ESTRIPEAUT-BOURJAC, 2012: 60). Par ailleurs, ESTRIPEAUT-BOURJAC (2012: 398) définit l' « écriture de l'urgence » comme une «[...] pratique testimoniale effectuée dans un contexte paroxystique ». Cette forme de représentation de l'histoire immédiate pratiquée dans un contexte d'urgence nous semble bien correspondre à la pratique de l'écriture post-sismique en Haïti, c'est-àdire les récits publiés après le tremblement de terre de janvier 2010. Dans les deux 
cas, haïtien et latino-américain, nonobstant son caractère hétérogène, l'écriture testimoniale vise à un objectif bien précis : informer et communiquer dans une situation d'urgence en défiant le «[...] contrôle institutionnel et officiel de l'Histoire et de la mémoire »(ESTRIPEAUT-BOURJAC, 2012: 56) ${ }^{3}$. Dans le cas haïtien, l' " écriture de l'urgence » est sous-tendue par le « devoir de mémoire » étant donné que la nécessité de témoigner dans l'urgence s'accompagne de la volonté de rendre hommage aux victimes et de transmettre un savoir sur les catastrophes, qu'elles soient historiques (comme l'oppression dictatoriale pendant le régime de François Duvalier) ou naturelles.

\section{Retour au témoignage et au "devoir de mémoire »}

Le tremblement de terre de janvier 2010 marque une date charnière qui met Haïti, la patrie meurtrie, de nouveau au-devant de la scène. Le contexte de l'aprèsséisme a fait rebondir le témoignage, perçu par plusieurs auteurs-survivants comme un impératif du « devoir de mémoire ». Cette " écriture de l'urgence» se trouvera à l'opposé du récit officiel sur la catastrophe qui se répand dans les médias internationaux. En réponse au silence du gouvernement haïtien, la littérature comme espace de médiation sera le seul moyen d'offrir une autre lecture de l'événement traumatique.

Selon le sociologue haïtien Laënnec HURBON (2014: 226), le silence du président de la République haïtienne, René Préval, a représenté une «abdication quasi totale de l'État », qui n'a pas assumé ses responsabilités dans la gestion de l'après-séisme. Les écrivains ont été ainsi les premiers à élever leur voix face à la catastrophe, poussés par un impératif éthique d'abord. Comme le souligne MameFatou NIANG (2013: 189), « [...] le séisme et ses répliques médiatiques installent le pays au cœur de la planète, et y braquent les regards du monde. Pour les écrivains et artistes haïtiens, l'enjeu est de profiter de cette plateforme mondiale afin de véhiculer leurs visions, leurs mots et dire leur île au monde ». Pour ces écrivains, dont certains ont vécu le cauchemar du séisme, témoigner consiste à écrire en contre-pied de l'objectivité présumée par les médias et par tout communiqué officiel $\mathrm{du}$ gouvernement. On peut même parler à cet égard d'un contre-discours, de «counter-narratives» (BUTTON, SCHULLER, 2016:4). Des auteurs haïtiens du Québec et en même temps personnalités publiques, comme Dany Laferrière et Rodney Saint-Éloi, ont vécu le séisme, étant retournés en Haïti pour participer au festival culturel «Étonnants voyageurs ». Ils ont par la suite communiqué leurs expériences et réflexions.

Martin Munro remarque que la plupart des romanciers, bénéficiant déjà d'une reconnaissance internationale avant l'événement, ont préféré témoigner au lieu de choisir le roman ${ }^{4}$. Beaucoup plus que de simples témoignages individuels, ces textes confirment la dimension collective de l'engagement. Anny Dayan

\footnotetext{
${ }^{3}$ Mark D. ANDERSON (2011 : 107-189) aussi a étudié l'emploi d'une forme d' « écriture de l'urgence » non-fictionnelle comme la crónica, largement utilisé au Mexique pour critiquer la situation sociopolitique du pays après le tremblement de terre qui a frappé la capitale mexicaine en 1985.

${ }^{4}$ Les auteurs cités par Munro $(2014$ : 22) sont Edwidge Danticat, Rodney Saint-Eloi, Yanick Lahens, Gary Victor et Dany Laferrière.
} 
ROSENMAN (2007: 23) souligne à cet égard que «[c']est dans l'énonciation individuelle du désastre collectif que s'inscrit l'importance cruciale que revêt le témoignage comme acte de transmission et comme acte d'écriture ». Avant d'interroger de près le récit-témoignage écrit par Rodney Saint-Éloi, notons que l'écriture de l'urgence relève d'une intention implicite. Mame-Fatou NIANG (2017 : 34-35) l'explicite en ces termes : "Le traitement littéraire du séisme du 12 janvier 2010 répond en priorité à quatre impératifs : honorer la mémoire des disparus, saluer la résilience des survivants, rectifier les discours biaisés sur le pays, et enfin, fournir dans la foulée du drame des propositions qui peuvent aider à sortir Haïti de l'ornière ». De la sorte, le témoignage effectue le passage de la position de "victime» et de témoin oculaire passif à «acteur» et témoin historique (DUSAILLANT-FERNANDES, 2014 : 18). L'écrivain-témoin - et les écrivains haïtiens ne font pas d'exception - doit souvent assumer une triple obligation morale : parler de sa propre expérience, des proches qui sont ou ne sont plus en vie, et de la dimension collective du désastre auquel il a survécu. Dans le cas haïtien, le "récit de soi » est le plus souvent un « récit sur soi » et sur les autres. Il s'agit, pour le survivant, de faire face à l'épreuve traumatique autant au niveau individuel que collectif.

Rodney Saint-Éloi, né en 1963 à Cavaillon, dans le sud du pays, a quitté Haïti en 2001 pour Montréal, où il a fondé la maison d'édition Mémoire d'encrier. Membre de l'Académie des lettres du Québec, auteur de récits et poèmes, éditeur et universitaire, Saint-Éloi joue un rôle central dans la vie culturelle montréalaise, où il est connu pour son engagement. Comme l'explique Thomas C. SPEAR dans la page consacrée à l'auteur sur le site Île en Île : «Chez Rodney Saint-Éloi, tout est engagement : écriture et édition. Engagement envers le social, engagement envers la littérature, engagement enfin vers tout ce qui libère ». Quand un tremblement de terre de magnitude 7,3 frappe Port-au-Prince, à 16 h53 le mardi 12 janvier 2010, Rodney Saint-Éloi et Dany Laferrière sont attablés au restaurant de l'hôtel Karibe. Trois mois après le séisme, paraît le récit de Dany Laferrière Tout bouge autour de moi. Saint-Éloi publie ensuite Haïti kenbe là! 35 secondes et mon pays à reconstruire, un récit-témoignage en partie autobiographique, où il fait une chronique des jours après la catastrophe.

Du point de vue de la structure de l'ouvrage, la narration n'est pas linéaire et la chronique de l'événement est seulement un aspect de ce témoignage hybride. Le texte a aussi une dimension méditative et la narration des faits s'arrête par moments pour privilégier une attitude plus contemplative. Ces séquences s'apparentent au genre de l'essai-méditation, défini par Marc ANGENOT (2003 : 155) comme « [...] une pensée "en train de se faire", à la recherche d'une conceptualisation, prise dans la langue du vécu, parfois balbutiante, parfois fulgurante ». À partir de son regard, de ce qu'il observe, Saint-Éloi est amené à réfléchir sur la situation haïtienne, quoique l'auteur donne une importance centrale aux souvenirs de son enfance passée en Haïti qui refont surface. C'est comme si ces souvenirs lui venaient en aide dans des circonstances qui se révèlent dures à accepter, au point que le matin après le séisme, au réveil, il admet qu'il préférerait tout oublier : « La seule chose dont je me souvienne c'est que je ne veux plus avoir de souvenirs » (SAINT-ÉLOI, 2010 : 119). Il évoque ainsi les soirées passées dans la cour de la maison, au début des 
années 1970, à écouter les contes de sa grand-mère, de qui il a appris ses premières leçons d'histoire. Il retrace le passé dans une longue séquence consacrée à l'arrivée de Christophe Colomb, « le premier séisme » (SAINT-ÉLOI, $2010: 202$ ) qui a atteint Haït, auquel ont fait suite d'autres secousses sismiques : «[...] l'histoire du pays depuis l'indépendance est une suite de séismes suivis de répliques régulières » (SAINT-ÉlOI, 2010 : 215). La méditation individuelle est immédiate et nécessaire, car « [...] dans un désastre général, il y a toujours le désastre de soi » (SAINT-ÉLOI, 2010 : 38). Pourtant, la réflexion proposée ne reste jamais enfermée ou purement subjective car elle est toujours conçue pour la collectivité. D'ailleurs, le titre en créole, Haïti kenbe la!, est un appel adressé au pays et au peuple haïtien : « Haïti, redresse-toi!»».

La situation d'énonciation correspond à la déambulation urbaine d'un individu naufragé. Saint-Éloi sillonne les rues de la capitale, en compagnie des écrivains Dany Laferrière et Lyonel Trouillot: ils traversent les quartiers de Pétionville, Delmas et Canapé-Vert jusqu'au Caribbean Market, un grand supermarché complètement effondré. Dans un passage saturé de références intertextuelles, l'auteur compare la ville dévastée à des descriptions post-apocalyptiques dans des romans comme L'Aveuglement de José Saramago, la Trilogie new-yorkaise de Paul Auster et La route de Cormac McCarthy. Pourtant, il constate que la réalité qui l'entoure est beaucoup plus cruelle que sa version fictionnelle. La sensation d'être au milieu de nulle part, de vivre l'anéantissement absolu de tout, est étayée par un sentiment d'abolition de tout repère temporel :

Télescopage de temps. Présent et passé. Personne ne sait plus si le présent est un passé décomposé. Tout le monde a la certitude que le présent est un prétexte à la catastrophe. Une mauvaise plaisanterie a sapé les fondamentaux : la terre, l'eau, l'air, le feu. Les éléments se sont mués en corps flottants, fragiles et inconstants. Le temps est catapulté dans un navire à la dérive. L'imparfait ronge le présent. Se rétracte le futur dans un amas boueux. La parole perd l'assise du temps. Hier. Aujourd'hui. Demain. Rien n'a de consistance. C'était une maison. C'était un jardin. C'était une famille. Maintenant que tous les membres de ladite famille ne répondent plus à l'appel, peut-on encore parler de famille ? La mère tient entre ses bras ouverts l'enfant mort. Est-ce un enfant ou un cadavre ? Le temps glisse, aveugle, dans l'imprécise virtuosité du désordre. (SAINTÉLOI, $2010: 71$ )

Saint-Éloi perçoit aussi les premiers signes de la vie au milieu des décombres. La force de la nature et sa vitalité font fi de ce décor d'apocalypse : « Dans ce silence grave, plein d'agonie, j'ai regardé les lilas du jardin, encore beaux. Tout laisse croire que le saccage ne les concerne pas. [...] Les plantes et les fleurs ont encore une fois montré leur supériorité en restant debout, impassibles, au milieu des cris et des émois » (SAINT-ÉLOI, 2010: 131-132). Les lilas déclenchent un double mouvement: en les observant, Saint-Éloi plonge d'abord dans les souvenirs d'enfance, ensuite il se rappelle un poème de l'haïtien Davertige, "Ombarigore ", où la nature est protagoniste. Nature et poésie sont évoquées pour leur pouvoir curatif : « À cette minute tragique, pour colmater les fissures, il y a eu en moi : le parfum des lilas et le poème de Davertige » (SAINT-ÉLOI, 2010: 134). Dans une autre section, en regardant les vivants, il remarque qu'ils « [...] sont debout tels des flamboyants » (SAINT-ÉLOI, $2010: 178$ ). Non seulement la nature, mais les gens aussi se redressent. Pour certains, le séisme représente une opportunité 
exceptionnelle, comme pour deux enfants des rues qui trouvent un cadeau inattendu - des fusils à eau - au milieu des débris :

Sous les décombres, se dégage un hymne à la vie réaffirmant sans cesse Se lavi. C'est la vie. Deux petits garçons cocorats, cette horde d'orphelins lâchés sur la ville, ces enfants du hasard et de l'ombre qui vivotent en rats. Nés dans des égouts, ils grandissent en marge de la vie, se lèvent au soleil levant avec pour seule mission le repas du jour. Aujourd'hui, quelle journée les attend! Alors qu'ils étaient en train de fouiller dans les décombres d'une maison, ils ont découvert deux fusils à eau, le cadeau de leur rêve. Le séisme a sur son compte un petit bonheur d'occasion. (SAINT-ÉLOI, $2010: 64$ )

Dans un autre passage, Saint-Éloi intègre dans son raisonnement l'intervention du journaliste Yves-Marie C. lors d'une émission à la station de radio Mélodie FM. Dans son bilan du séisme, le journaliste souligne les opportunités que le pays devrait saisir pour se redresser et il donne la priorité à la reconstruction : "Le séisme est peut-être une chance pour grandir et pour mieux regarder en nous. Pour voir grand. Les écoles n'existent plus. Il faut de nouvelles écoles avec une nouvelle vision. De grandes écoles. De grandes cours. De grands professeurs. De grandes salles. De grandes ambitions. Des écoles grandioses pour l'avenir »(SAINT-ÉLOI, 2010 : 175).

\section{Le « sentiment de double appartenance » de l'auteur émigré}

L'importance accordée à la méditation de l'auteur sur sa condition d'émigré et sur sa situation diasporique est ce qui distingue Haïti kenbe là ! des autres récitstémoignages post-sismiques. Le départ de Saint-Éloi d'Haïti pour Montréal a été tardif, à l'âge de 42 ans. Dans son récit, il parle explicitement d' " exil » (SAINTÉLOI, 2010 : 32) et il se considère comme un « exilé » (110) : « J'étais à Montréal, en plein exil, loin de moi-même » $(98) ;[\ldots]$ l'exil est une profession de foi dans la douleur et dans le manque, où que l'on soit, ici ou ailleurs, la douleur nous rattrape » (99). Le «sentiment de double appartenance » dont parle Yanick LAHENS (1990 : 57) semble caractériser le rapport de l'auteur avec les deux pays entre lesquels il est tiraillé. Cela frappe dès les premières observations, où il décrit les sensations ressenties après avoir atterri à Port-au-Prince, le 12 janvier 2010 :

Entrer en moi-même, me replacer dans ma vie d'avant, me fondre dans ces paysages que j'ai laissés il y a dix ans pour aller vivre à Montréal. Vivre dans l'aller-retour, entre deux pays, dans un ballottage continu, le tourment d'être une girouette entre deux terres, le corps et le cour coupés en deux. Vivre surtout longtemps avec la mauvaise conscience d'être si loin et si peu présent dans le combat pour le changement au pays natal. C'est le prix que tout exilé doit payer. (SAINT-ÉLOI, $2010: 31-32)$

Ce genre de réflexion renvoie à la définition d' « exilé » donnée par LAHENS (1990 : 15), selon qui « [ê]tre exilé, c'est être d'ici et d'ailleurs, c'est être à la fois dedans et dehors, installé dans l'insécurité d'un porte-à-faux douloureux et malaisé ». Saint-Éloi semble incarner parfaitement la condition existentielle de l'émigré pour qui la question de l'entre-deux est encore ouverte. Un exemple saillant est le fait qu'il n'a pas encore démarré la procédure pour l'obtention de la nationalité canadienne : "Cela fait longtemps que je dois troquer mon passeport contre un passeport canadien. À l'ambassade et dans les aéroports, je me fais toujours agresser 
avec les mêmes questions : pourquoi, après tout ce temps, n'as-tu pas la nationalité canadienne? Quand vas-tu remplir les formulaires de naturalisation? » (SAINTÉLOI, 2010 : 135-136).

Selon Martin MunRo (2014 : 43), le fait d'être resté plus longuement en Haïti par rapport à Dany Laferrière après le tremblement de terre aurait été bénéfique pour Saint-Éloi, car cela lui aurait permis d'avancer dans le processus de perlaboration du traumatisme et, par la suite, de mieux accéder aux souvenirs de l'événement et de les comprendre. Il faut pourtant constater que, dans l'immédiat, Saint-Éloi semble être tourmenté par sa double identité d'haïtiano-québécois. Les secousses dévastatrices déclenchent chez lui une méditation sur le sens du mot "patrie » et celui de l'action de " rapatrier ", après avoir assisté au rapatriement des citoyens canadiens, parmi lesquels son ami Laferrière, car ce qui compte pour les militaires est le passeport : « La feuille d'érable, ça passe... Le palmiste, ça reste. Rapatrier... cela paraît drôle. Ma patrie, c'est bien ici, dans cette terre à séisme » (SAINT-ÉLOI, 2010 : 136). Le retour en Haïti, sa patrie, a toujours été vécu par l'auteur comme un retour aux origines, aux parfums et aux souvenirs d'un passé heureux : " Je rentre en Haïti, au pays de l'enfance, je suis déjà submergé par les effluves de citronnelle, de cannelle, de vétiver et de camomille. Il ne faut jamais laisser derrière soi son enfance. Surtout en hiver, il est bon d'y retourner pour mieux voir en soi sur les lieux. Dresser l'inventaire de son enfance est la meilleure chose qui puisse arriver à un exilé... » (SAINT-ÉLOI, 2010 : 109-110).

Dans son analyse du texte de Saint-Éloi, Martin MunRo (2014 : 45) constate que le tremblement de terre a changé pour toujours la perception de l'auteur par rapport à sa condition d'exilé qui rentre au pays natal. Après le séisme, Saint-Éloi comprend qu'il ne pourra jamais habiter à nouveau son passé, car le paysage a visiblement changé dans son aspect concret :

Que devient-on quand on retourne chez soi, qu'on est propulsé sans transition sur un court de tennis avec trois cents personnes? C'est peut-être cela le retour au pays natal, un voyageur/retourneur nu, sans bagages, sans histoire, avec la mémoire pour héritage, et l'angoisse, celle-là ferme, de la terre qui a tremblé. En trente-cinq secondes, tout change pour chacun d'entre nous, et pour le pays. (SAINT-ÉLOI, $2010: 37-38$ )

Pour celui qui ne peut plus trouver de repères, la seule possibilité est de faire appel à la mémoire, pour retrouver ce qu'il appelle le "royaume de l'enfance » (SAINT-ÉlOI, $2010: 196)^{5}$. Cela permet de mettre à distance, ne serait-ce que pour un moment, l'épreuve du désastre.

\section{La « faille diasporique » et la création littéraire}

Le mot polysémique «faille» se réfère d'abord, dans le contexte de l'aprèsséisme, à la faille géologique, cause immédiate du tremblement de terre, mais aussi à de nombreuses failles historiques, sociales, économiques et linguistiques qui caractérisent la société haïtienne et qui ont été brutalement dénudées par la violence du séisme. La faille a aussi une connotation temporelle ; elle signifie une déchirure

\footnotetext{
${ }^{5}$ Pour une analyse du rapport entre histoire et mémoire dans le texte de Rodney Saint-Éloi, voir Kasia MiKA (2014).
} 
dans l'ordre naturel des choses, une fracture qui a englouti les souvenirs et le passé des Haïtiens en signalant une rupture entre l'avant et l'après. Dans le texte de SaintÉloi comme dans le récit-témoignage Failles (2010) de Yanick Lahens, on remarque un aspect commun : la proximité entre la faille géologique extérieure, concrète, et une faille intérieure, de celui qui a survécu à la catastrophe. Comme le constate Yves ChemLa (2015:231), « [...] la faille qui a morcelé les paysages et englouti les êtres s'est aussi creusée dans l'intime ». Cette faille intime, invisible mais toujours lancinante, est un défi pour l'écrivain : « À chaque réplique, j'ai appris à taire en moi les larmes pour faire semblant d'être plus fort que le séisme. Chaque réplique me rapprochait de la faille, de ma faille, confirmant que cette terre ramollie, limon, bourbe, était désormais capable de s'en aller sous nos pieds » (SAINT-ÉLOI, 2010: 245). Chez Saint-Éloi, on aperçoit non seulement un bouleversement du paysage extérieur et du paysage intime, mais aussi l'omniprésence du « sentiment de double appartenance » que le séisme semble avoir renforcé, voire exacerbé. Aux failles déjà mentionnées, s'ajoute chez Saint-Éloi une autre faille, que nous pourrions appeler « diasporique ».

L'apparition de cette faille est visible dans quelques passages d' Haïti kenbe là ! qui se situent du point de vue chronologique après le retour de l'auteur à Montréal. Seule la création littéraire semble pouvoir constituer un remède contre la déchirure éprouvée par l'écrivain haïtiano-québécois, amplifiée par le désastre. L'auteur décrit par ces mots son arrivée dans la ville québécoise, quelques jours après le séisme : « J'atterris à Montréal à l'aéroport Pierre-Elliott Trudeau le lundi 18 janvier vers 5 heures du matin. [...] J'ai une idée fixe : rentrer chez moi. Chez moi, c'est cet appartement de la rue Chabot dans le quartier Rosemont, un magnolia près de l'escalier colimaçon en fer forgé, le silence des souvenirs, les toiles sur les murs et les livres qui attendent sur des étagères en bois » (SAINT-ÉLOI, $2010: 237-238$ ). Sur la route qui mène de l'aéroport à sa maison, Saint-Éloi, en observant la neige et la lumière de l'aube montréalaise, pense à un poème du québécois Gérald Godin, « Pays », en particulier à ces quelques vers qui parlent du difficile « chez soi » du poète québécois : « J'ai vu le soleil se lever/ dans tant et tant de pays / je ne savais plus lequel / était le mien » (SAINT-ÉLOI, 2010 : 238). Encore une fois, la poésie lui vient en aide dans les moments qui ont fragilisé son identité haïtienne.

Une fois rentré à Montréal, Saint-Éloi se rend bientôt compte que le retour à la normalité, à sa vie d'avant le séisme, n'est pas possible : « À Montréal, je suis déjà réinstallé dans ma vie d'avant. Mais le goudou-goudou 6 me poursuit. Quand sonne le téléphone, ce sont des camarades qui appellent du pays pour donner des nouvelles, pour faire la chronique des secousses. Je sens dans leur voix le cri à peine étouffé, qui gronde entre les mots, et qui martèle les sons du goudou-goudou » (SAINT-ÉLOI, 2010 : 246-247). Les images à la télé, les nouvelles diffusées par la radio, les amis et les voisins qui lui rendent visite ou qui l'appellent, tout rappelle à cet Haïtien de Montréal qu'il lui est impossible d'échapper à la catastrophe: «À Montréal,

\footnotetext{
${ }^{6}$ L'expression « goudou-goudou » est une onomatopée utilisée par les gens des quartiers populaires pour se référer au tremblement de terre. Comme l'explique Nancy DorsinviLle (2012 : 292-293), elle serait une transcription en créole du son entendu au moment des secousses, provenant des entrailles de la terre.
} 
comment aurais-je pu suivre de loin sur le petit écran ces images sans mourir dans chaque mort? » (SAINT-ÉLOI, $2010: 265$ ).

Après avoir été le témoin d'un événement catastrophique, le survivant peut vivre dans un état de stress permanent, souvent pathologique, comme l'explique Yoann MOREAU (2017 : 58) : « Sous l'effet d'un fort traumatisme, les victimes sont sujettes à des réminiscences, leur attention tourbillonne en permanence, reproduisant physiquement et mentalement les scènes de la catastrophe. Elles vivent dans leur chair le moment du désastre comme s'il était encore d'actualité ». Dans certains passages du texte, les effets du traumatisme et le stress post-traumatique dont l'auteur souffre sont explicités : «Il est 3 heures du matin. J'éteins la lumière. La chambre n'a pas d'issue. La fenêtre est bien trop haute. Le plafond valse. Le sol s'ouvre avec des cris. Des prières. Des cadavres. Une fillette danse entre deux rangées de lilas. Des murs tombent. Des chaises rebondissent. La terre bascule. L'ombre descend sur toute chose. L'horreur est en moi » (SAINT-ÉLOI, 2010 : 243). Des hallucinations hantent le rescapé, comme des flashback de la catastrophe, même en plein jour, et la chambre de sa maison à Montréal devient une prison où se lovent les images de la catastrophe. Le rôle de la littérature est alors évoqué : « La nuit, je me sens balancé. Le plafond descend sur moi. La terre vacille au moindre mouvement. Je me mets à lire ou à écrire pour oublier que la terre, qui sait nourrir, peut aussi trembler et tuer » (SAINT-ÉLOI, $2010: 267$ ). La pratique de l'écriture est dès lors conçue comme un moyen de donner sens à l'événement en le traduisant en mots, pour entamer ainsi un travail de deuil thérapeutique, afin de ne pas sombrer dans la folie ou le désespoir : «J'ai écrit ce livre assurément pour faire taire en moi les fureurs du goudou-goudou » (SAINT-ÉLOI, $2010: 267$ ).

\section{Conclusion}

Conformément à l'un des critères fondamentaux qui définissent le genre testimonial, le devoir de l'écrivain-témoin, au-delà de son aspect individuel, répond toujours à une nécessité collective, destinée à toute la communauté de survivants : «J'ai écrit ce livre pour accompagner d'une berceuse ce cri goudou-goudou enraciné dans les entrailles de tous les Haïtiens. C'est une blessure avec laquelle ils seront obligés de vivre » (SAINT-ÉLOI, 2010 : 266). La transmission d'un savoir sur le désastre est une étape essentielle qui permet la création d'une "culture de la catastrophe », comme le souligne le Suisse Peter UTz $(2017,387)$ : « On raconte des catastrophes et on en racontera toujours, car c'est un des moyens les plus infaillibles de les surmonter tout en les intégrant dans la culture $»$.

Avec son témoignage, Rodney Saint-Éloi accomplit son devoir de mémoire en exposant toutes les difficultés vécues par l'émigré tiraillé entre sa patrie et le pays d'accueil, " entre l'ancrage et la fuite » pour rappeler le titre de l'essai de Yanick Lahens. En ce sens, l'expérience tragique vécue par l'auteur aura intensifié un « sentiment de double appartenance » latent, et aura mis en relief l'existence d'une « faille diasporique » que seule la pratique d'écriture a le pouvoir de colmater. 


\section{BIBLIOGRAPHIE}

ANDERSON Mark D. (2011), Disaster Writing: The Cultural Politics of Catastrophe in Latin America, Charlottesville, University of Virginia Press.

ANGENOT Marc (2003), Remarques sur l'essai littéraire, in : DUMONT François (éd.), Approches de l'essai : anthologie, Québec, Éditions Nota bene.

BUTTON Gregory V., SCHULlER Mark (2016), Introduction, in : BUTTON Gregory V., SChUller Mark (éds.), Contextualizing Disaster, New York/Oxford, Berghahn Books, p. 1-16.

CHEMLA Yves (2015), Littérature haïtienne 1980-2015, Delmas, C3 Éditions.

DES Rosiers Joël (2013), Métaspora : essai sur les patries intimes, Montréal, Triptyque.

DORSINVILle Nancy (2012), Goudou Goudou, in : FARMER Paul, Haiti After the Earthquake, New York, PublicAffairs, p. 285-293.

DUSAILlANT-FERNANDES Valérie (2014), Quand un tremblement de terre secoue l'écriture : regards croisés sur une catastrophe dans Tout bouge autour de moi de Dany Laferrière et Failles de Yanick Lahens, in : VANBORRE Emmanuelle Anne (éd.), Haïti après le tremblement de terre : la forme, le rôle et le pouvoir de l'écriture, New York, Peter Lang, p. 15-29.

ESTRIPEAUT-BOURJAC Marie (2012), L'Écriture de l'urgence en Amérique Latine, Pessac, Presses Universitaires de Bordeaux.

HURBON Laënnec (2014), L'État haïtien face à la catastrophe du 12 janvier 2010, in : Hurbon Laënnec (éd), Catastrophes et environnement. Haïti, séisme du 12 janvier 2010, Paris, Éditions de l'EHESS, p. 219-241.

KWATERKO Józef (2002), Les fictions identitaires des romanciers haïtiens du Québec, Revue de littérature comparée 302 (2), 2002.

LAHENS Yanick (1990), L'Exil : entre l'ancrage et la fuite, l'écrivain haïtien, Portau-Prince, Éditions Henri Deschamps.

LAHENS Yanick (2010), Failles, Paris, Sabine Wespieser Éditeur.

LALIEU Olivier (2001), L'invention du « devoir de mémoire », Vingtième Siècle, revue d'histoire 69, p. 83-94.

MIKA Kasia (2014), Histories of the Past, Histories for the Future : Representing the Past and Writing for the Future in Rodney Saint-Éloi's Haïti kenbe la !, Journal of Haitian Studies 20 (2), p. 4-19.

MOREAU Yann (2017), Vivre avec les catastrophes, Paris, PUF.

MUNRO Martin (2014), Writing on the Fault Line: Haitian Literature and the Earthquake of 2010, Liverpool, Liverpool University Press.

NiANG Mame-Fatou (2013), Kenbe la ! Haïti ou l'écriture de l'urgence, in : EL Nossery Névine, HubBel Amy L. (éds.), The Unspeakable : Representations of Trauma in Francophone Literature and Art, Newcastle upon Tyne, Cambridge Scholars Publishing, p. 173-192.

NIANG Mame-Fatou (2017), Il était une fois une ville : pratiques et poétiques de l'espace dans le Port-au-Prince de l'après 12 janvier 2010, in : SATYRE Joubert (éd.), Horizons multiples de l'écriture haïtienne contemporaine, Montréal, CIDIHCA, p. 17-39. 
Rosenman Anny Dayan (2007), Les alphabets de la Shoah. Survivre. Témoigner. Écrire, Paris, CNRS Éditions.

SAINT-ÉLOI Rodney (2010), Haïti kenbé la!, Neuilly-sur-Seine, Michel Lafon.

SPEAR Thomas C., Rodney Saint-Éloi, Île en île [en ligne], http://ile-enile.org/saint-eloi/ [consulté le 03/11/2019].

UTZ Peter (2017), Culture de la catastrophe, les littératures suisses face aux cataclysmes, Carouge-Genève, Éditions Zoé.

VALliÈres Pierre (1968), Nègres blancs d'Amérique, autobiographie précoce d'un " terroriste » québécois, Montréal, Éditions Parti Pris. 